Review

\title{
Emerging Role of Tumor-associated Macrophages as Therapeutic Targets in Glioblastoma Multiforme
}

\author{
Xian-Bin Lin ${ }^{1}$, Guo-Han $\mathrm{Hu}^{1,}$, , Hua-Lin $\mathrm{Fu}^{2}$, Wei-Lin Jin ${ }^{2,3,}$ \\ ${ }^{1}$ Department of Neurosurgery, Shanghai Changzheng Hospital, Second Military Medical University, Shanghai 200003, China \\ ${ }^{2}$ Key Laboratory for the Genetics of Developmental and Neuropsychiatric Disorders, Bio-X Institutes, Ministry of Education; \\ Institute of Nano Biomedicine and Engineering, Shanghai Jiao Tong University, Shanghai 200240, China \\ ${ }^{3}$ School of Life Sciences and Biotechnology, Shanghai Jiao Tong University, Shanghai 200240, China
}

\Corresponding author: E-mail: huguohan6504@sina.com; weilinjin@yahoo.com or weilinjin@sjtu.edu.cn

Received: March 3, 2014; Accepted: March 18, 2014; Published: March 20, 2014.

Citation: Xian-Bin Lin, Guo-Han Hu, Hua-Lin Fu and Wei-Lin Jin. Emerging Role of Tumor-associated Macrophages as Therapeutic Targets in Glioblastoma Multiforme. Nano Biomed. Eng. 2014, 6(I), 7-I8.

DOI: 10.5101/nbe.v6il.p7-18.

\begin{abstract}
The most common types of primary brain tumors in adults are gliomas. Glioblastoma multiforme (GBM) is the most highly aggressive type of glioma. GBM contains various numbers of cells with characteristics of activated or dysmorphic macrophages/microglia. Among them, some cell types provide significant support for tumor growth, while others are able to inhibit tumor progression. These cells are generally considered part of the tumor stroma and are often described as TAMs (tumourassociated macrophages). The presence of TAMs has been linked to increased tumor grade and poor clinical outcome in GBM, suggesting that depletion or inhibition of these cells may suppress tumor growth. A better understanding of tumor microenvironment in the brain would therefore be expected to contribute to the development of improved therapies for brain tumors that are urgently required due to a poor availability of treatments for these malignancies. This review summarizes some of the known interactions between brain tumors and different stromal cells, and also discusses potential therapeutic approaches within this context.
\end{abstract}

Keywords: GBM; TAMs; microenvironment; Nanoprodrug; immunosuppressive

\section{Introduction}

Malignant gliomas are the most common type of primary brain cancer while patients' median survival is approximately 15 months even with aggressive therapy [1-3]. Gliomas that are classified as grade I, II, III and grade IV (also referred to as Glioblastoma multiforme, GBM) according to histopathological criteria defined by the World Health Organization
(WHO). GBM is characterized by uncontrolled

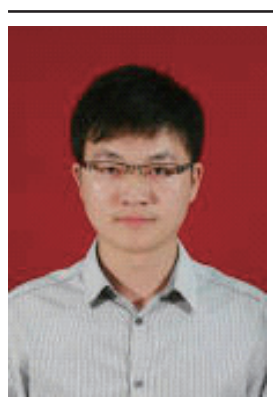

Mr. Lin Xianbin did his undergraduate study in Xiamen University from 2007 to 2012 . In 2012, He entered the Second Military Medical University with major in neurosurgery. He is now affiliated with the department of Neurosurgery, Shanghai Changzheng Hospital, Second Military Medical University, Shanghai, China. 
proliferation, areas of necrosis and diffuse infiltration [4]. GBM is one of the most lethal cancers. Malignant gliomas represent approximately $80 \%$ of all malignant brain tumors accounting for as many as 26,000 U.S. and European deaths annually, making them a significant unmet medical need [5]. There has been no major improvement in survival rate of glioma patients for the past several decades. Current standard treatment of GBM is surgical resection of the tumor, followed by adjuvant radiotherapy and chemotherapy [6]. Complete surgical resection of GBM is extremely rare. Radiation therapy, which enhances the necrotic microenvironment, often results in further tissue damage and more aggressive tumors. Since surgery, radiotherapy, chemotherapy, or a combination of multiple modalities cannot achieve satisfactory treatment outcomes, the development of effective therapeutic options on all scientific and clinical fronts to improve GBM patient outcome is desperately necessary [7].

The main challenge for successfully treating GBM is its diffuse infiltration of the adjunction brain parenchyma that renders the tumor cells refractory to surgery, chemo-radiotherapy and immune surveillance, leading invariably to recurrence [8]. The aggressive invasion by malignant glioma cells into surrounding normal brain tissues has increasingly been recognized as an important cause for frequent relapse that leads to high mortality [9-10]. Therefore investigation of the mechanism of glioblastoma cell invasion has received a great deal of interest. Development of novel therapeutic modalities is hampered by the lack of reliable prognostic markers and molecular targets. Despite extensive research, treatment options for malignant gliomas remain limited. Although GBMs are immunogenic tumors, they are not eradicated by the immune system [11]. While immunotherapeutic approaches have demonstrated safety and promising preliminary activities, their effectiveness can be improved by overcoming the immuno-suppressive mechanisms induced by these tumors [2, 12]. Indeed there is a growing interest in establishing novel immunotherapeutic approaches to the management of GBM patients.

One difficulty in treating GBM is substantial tumorcell and genetic heterogeneity, leading to aberrant activation of multiple signaling pathways [13-15]. In contrast, noncancerous stromal cells in the tumor microenvironment are genetically stable therapeutic targets [16-17]. The contemporary anti-tumor strategies are shifting from tumor-cell centric to those targeting various components of the tumor microenvironment [18-19]. The tumor microenvironment is a mixture of extracellular matrix molecules, tumor cells, endothelial cells, fibroblasts, and immune cells [20-21]. Among the tumor-infiltrating leukocytes, the predominant infiltrating cells comprise macrophages/microglia, which are referred to as tumor-associated macrophages (TAMs). TAMs can contribute to one-third or more to their actual tumor mass [22-23]. The molecular and functional characterization of TAMs is a current topic of investigation, and the interplay between TAMs and neoplastic cells represents a promising target for future therapeutic approaches.

\section{Tumor-Associated Macrophages are in charge of the tumor micro- environment}

TAMs not only stimulate the survival, proliferation, invasion and metastasis of tumor cells, but also construct a supportive stroma by promoting tumor vascularization and suppressing antitumor immunity by secreting growth factors, immune-suppressive cytokines and angiogenic factors and metalloproteinase [24]. The mechanism of this macrophage recruitment into viable glioblastomas is far from clear. The functional role of these tumour-infiltrating macrophages is also largely unknown. Of note, one recent study suggests that TAMs, arising from peripheral monocytes rather than resident microglia, are the predominant inflammatory cells to infiltrate human glioma [9].

Interestingly, there are two macrophage subpopulations that play separate roles in tumorigenic processes. Macrophages can become classically activated (M1 activation) in response to pathogens and pro-inflammatory molecules, resulting in killing microorganisms and tumor cells and produce copious amounts of proinflammatory cytokines and antigen processing and presentation [25-28]. Classically activated M1-type macrophages participate in the coordinated response to immunogenic antigens primarily through production of proinflammatory mediators (such as TNF- $\alpha$, IL-1B, and IL-12), upregulation of cell surface molecules necessary for antigen presentation (including MHC II and costimulatory molecules CD80 and CD86), and an overall enhanced ability to phagocytose pathogenic material [29-31]. 
In contrast, macrophage/microglia become immunosuppressive as designated as M2-type macrophages in response to anti-inflammatory molecules such as IL-4 (interleukin-4) and IL-10 and are unable to produce proinflammatory cytokines, induce effector T-cell anergy, demonstrate impairments in cytotoxicity, and induce Tregs because of their antiinflammatory functions [25, 32]. Tumor-associated M2 MFs have been shown to promote cancer by secreting proangiogenic factors and enhancing invasion mediated by the production of soluble factors such as transforming growth factor-b (TGF- $\beta$ ), interleukin (IL)-1, vascular endothelial growth factor, and matrix metallopeptidase. Alternatively activated M2-type macrophages do not secrete the proinflammatory mediators IL-1B or TNF- $\alpha$ and are believed to exert immunomodulation primarily through secretion of the potent immunosuppressive cytokines IL-10, IL-6, and TGF- $\alpha$, down regulation of cell surface molecules necessary for antigen presentation including $\mathrm{MHC}$ II, CD80, and CD86, decreased phagocytic capacity, and upregulation of cell surface antigens FasL and B7-H1 both known to stimulate programmed cell death in lymphocytes, among other effects [33-36]. Recent studies also showed that the presence of M2 macrophages is associated with a poor prognosis for patients with many kinds of tumor, including gliomas [22, 37-38]. Therefore, it is speculated that the inhibition of macrophage polarization toward the M2 phenotype could represent a new strategy for anticancer therapy.

\section{Features of Tumor-Associated Macrophages}

As previously mentioned, an increasing number of research indicates that M2-type of TAMs are in charge of the immunosuppressive function in the tumor microenvironment [39-40]. Besides, TAMs also act as the preponderant element included in the immune infiltration of human malignant glioma, finally result in facilitation of glioma cell progression. That's why it would be extremely important to have a better understanding about Tumor-Associated Macrophages.

\section{TAMs express immunosuppressive antigens and soluble mediators}

Recent reports have described that glioma TAMs reduce immunological responsiveness. Glioma TAMs are now thought to represent an altered phenotype resulting from the directed influence of tumor cells upon immune cells in efforts to turn into a suitable tumor microenvironment [27, 41-42]. As shown in a recent series of experiments, glioblastoma-conditioned monocytes dramatically increased immunosuppressive interleukin-10, transforming growth factor-beta, and B7-H1 expression, decreased phagocytic ability, and increased ability to induce apoptosis in activated lymphocytes [43]. Hence, expression of IL-10 by TAMs in glioma tissue appears to be an important immunosuppressive intermediary agent of the glioma microenvironment, and its subsequent expression in glioma cells may serve both as an initial agent to recruit monocytes, as well as the driver of feed forward loops of immunosuppressive mediator expression between tumor cells and macrophages within the tumor microenvironment [41, 44].

Badie et al. demonstrated that nearly every infiltrating monocyte-derived cell in murine glioma models expressed FasL [45]. FasL expression can stimulate programmed cell death in lymphocytes and also support the growth of experimental intracranial glioma [46]. Down-regulating FasL expression and/ or function in glial malignancies can enhance T-cell tumor infiltration and inhibit tumor growth [47]. This hypothesis is strengthened by the finding that neutralization of FasL results in a significant increase in the number of tumor-infiltrating lymphocytes in a murine glioma model [48]. The findings suggest that targeting endogenous FasL in glial malignancies could enhance the efficacy of emerging immune-based treatment strategies.

B7-homologue 1 (B7-H1), a recently identified homologue of B7.1/2 (CD80/86), has been described to exert costimulatory and immune regulatory functions through engagement of the programmed death receptor (PD-1) on the surface of $\mathrm{T}$ cells [49]. PD-1 activation on T cells by B7-H1 has been shown to initiate an intracellular signaling cascade resulting in downregulation of T-cell receptor (TCR) signaling and may also promote T-cell apoptosis $[50,51]$. Although the precise role of B7-H1 is still unknown, mutual expression of B7-H1 in both glioma and TAM cells may provide a critical mechanism by which lymphocyte suppression is achieved in the tumor microenvironment. These findings suggest that inhibition of classically activated antitumor effector functions of glioma M1-type TAMs may be a longlasting regulatory phenotype. 


\section{TAMs reduced antitumor function in glioma}

In addition to the immunosuppressive function described earlier, TAMs in malignant gliomas demonstrate a significant reduction in specific proinflammatory or antitumor function. Much of this is evidenced by studies showing reductions in secretion of proinflammatory cytokines and increases in secretion of inhibitory cytokines. The mature M1 macrophage marker CD14 serves as a coreceptor of TLR4 and is upregulated in almost all brain tumor [51]. However, downregulation of CD14 has been observed in TAMs in several other cancers, and Rodriguez and Parney et al. demonstrated that monocytes isolated from healthy subjects reduce CD14 (but not CD11b) expression upon exposure to human glioblastoma cell lines [43]. This represents another potential mechanism by which TAMs have diminished antiglioma activity.

Other deficiencies of glioma TAMs found to promote local immunosuppression are decreased in the expression of HLA and costimulatory molecules. Badie and Schartner who used FACS to demonstrate little to no expression of MHCII, CD80, or CD86 on macrophages freshly isolated from rat gliomas. Furthermore, expression of these costimulatory molecules on TAMs could not be restored by stimulation with IFN- $\gamma$ or LPS [52]. There is suppressed secretion of IFN- $\gamma$ from CD4+ $\mathrm{T}$ cells cultured with GBM-treated monocytes [41]. Similar evidence regarding downregulation of cell surface molecules and reduction of T-cell activation were reported by Rodrigues et al., demonstrating that following coculture with malignant glioma cells, human monocytes induce apoptosis in activated autologous $T$ cells [43].

TAMs have also been shown to be short of phagocytosis. Rodrigues and colleagues demonstrated a significant decrease in the ability to phagocytose bacterial cell wall particles following stimulation in glioma cell conditioned monocytes, as compared with both astrocyte conditioned and unconditioned monocytes [43]. In another report, Hussain et al. demonstrated active phagocytosis of opsonized beads in macrophages isolated from ex vivo human GBM specimens [32]. This group further attempted to show that TAMs isolated directly from human GBM tumors are deficient of non-MHC-restricted antitumor cellular toxicity through coculture with a target cell line derived from malignant human glioma [32]. Their results indicate minimal cytotoxic ability of these glioma TAMs, as compared to naive microglia isolated from normal brain tissue.

\section{TAMs expression of multiple glioma- promoting mediators: augmenting glioma progression}

Glioma cells could produce a number of self-supportive factors concurrently with their corresponding cell surface receptors, together acting to promote their own proliferation, migration, angiogenesis, and subsequently tumor extension [5356]. Indeed, current models of the glioma tumor microenvironment suggest a potent milieu of trophic and immunomodulatory factors bathing all tumor cells and propagating tumor growth through autocrine and paracrine loops of expression and stimulation [41]. Less clear than their lack of effector function or their expression of immunosuppressive mediators, glioma TAMs are increasingly implicated in the contribution of glioma-promoting tumor trophic factors to the local microenvironment. Among the tumor supportive factors potentially secreted by TAMs, TGF- $\beta$, EGF, and $\mathrm{HGF} / \mathrm{SF}$ have drawn the most attention, though dissecting the precise role of TAMs in the production of these trophic factors remains to be accomplished.

\section{Potential therapeutic targets}

\section{Flt-1}

Vascular endothelial growth factor (VEGF) is an requisite angiogenic growth factor to provide adequate blood supply in tumors. The biological feature of VEGF family members is mediated by the activation of tyrosine kinase receptors structurally related to fms/kit/ PDGFR family [57]. Several lines of evidence indicate that Flt-1, a fms-like tyrosine kinase receptor, which binds to vascular endothelial growth factor (VEGF)-A, VEGF-B, and PlGF (Placental growth factor) [58], is a positive regulator of angiogenesis in the context of tumor growth and metastasis. Except for endothelial cells, Flt-1 is also expressed by other different cell types, including monocytes and macrophages [59-60].

Flt-1 could be involved in macrophage-mediated tumor growth through at least two pathways: First, via Flt-1-kinase-dependent macrophage activation and expression of several proangiogenic factors; second, Flt-1 might be able to induce the migration of monocytes toward VEGF-A and PlGF [61-62]. Loss of 
Flt-1 signaling in tumor infiltrating macrophages has a remarkable suppressive effect on tumor growth and vascularization in an experimental glioma model [63]. Flt-1 signaling in macrophages has been implicated in promoting cell survival and migration. VEGF/Flt1 in macrophages might serve to amplify expression of proangiogenic factors, including VEGF itself [64]. Several groups have shown that Flt-1 is crucial for VEGF dependent monocyte/macrophage migration [63]. VEGF and PIGF overexpression by tumor cells induced an accumulation of bone marrow-derived myeloid cells into tumor site. This infiltration was decreased in tumors grown in Flt-1 TK-/-bone marrow chimeras [65-66].

Flt-1 signaling in macrophages might be able to amplify the expression of available VEGF in the glioma tissue and might represent an important step particularly in the initial phases of tumor growth [67]. Therefore, targeting Flt-1 receptor in bone marrowderived infiltrating macrophages might be a prospective approach towards the future antiangiogenic therapies in gliomas.

\section{CSF-1R}

Colony-stimulating factor 1 receptor (CSF1R) is involved in macrophage differentiation and survival. CSF-1R blockade slowed intracranial growth of patient-derived glioma xenografts. CSF$1 \mathrm{R}$ inhibition blocks glioma growth and progression through a mechanism in which TAMs are not depleted but are instead 're-educated' within the glioma microenvironment [13]. TAMs in all GBM models were specifically protected from CSF-1R inhibitorinduced death. This contrasted with the observed depletion of microglia in the normal brain and the depletion of macrophages in other tissues, which is consistent with previous reports [68-69]. Modeling these microenvironment-mediated effects in culture allowed us to identify GM-CSF and IFN- $\gamma$ as gliomasupplied factors that facilitate macrophage survival in the context of CSF-1R inhibition.

The new drug, called BLZ945, penetrates the brain and has high affinity and specificity for colonystimulating factor 1 receptor (CSF-1R), specifically reduced macrophage proliferation and survival in vitro without affecting glioma cell viability [24]. Its antitumor effect was mediated by decreased tumor cell proliferation and vascularity and increased apoptosis following BLZ945 treatment. However, BLZ945 did not promote depletion of TAMs, which were protected from CSF1R inhibition by glioma-secreted factors. In contrast, BLZ945 triggered downregulation of protumorigenic, alternatively activated M2 macrophage markers and enhanced the phagocytic function of glioma-derived TAMs, suggesting that CSF1R blockade-induced depolarization inhibits the tumor promoting activity of TAMs [13]. Indeed, BLZ945 suppressed glioma cell-TAM heterotypic signaling, resulting in decreased glioma cell proliferation. Moreover, gene signatures associated with BLZ945 treatment were predictive of increased overall survival in patients with proneural GBM, independent of macrophage numbers [70]. These findings suggest that re-education, rather than depletion, of macrophages in the tumor microenvironment, combining CSF$1 \mathrm{R}$ inhibition with standard-of-care therapies, such as radiation and temozolomide may provide more efficient therapeutic options for patients with GBM.

\section{TGF- $\beta 1-T G F B R 2$ pathway}

In primary human gliomas and orthotopical transplanted syngeneic glioma, the number of TAM/ $\mathrm{Ms}$ at the invasive front was correlated with the presence of CD133+ glioma stem-like cells (GSLCs), and these TAM/Ms produced high levels of TGF- $\beta 1$ [9]. CD133+ GSLCs isolated from murine transplanted gliomas exhibited higher invasive potential after being cocultured with TAM/Ms, and the invasiveness was inhibited by neutralization of TGF- $\beta 1$. It also has been found that human glioma-derived CD133+ GSLCs became more invasive upon treatment with TGF- $\beta 1$. In addition, compared with CD133- committed tumor cells, CD133+ GSLCs expressed higher levels of type II TGF- $\beta$ receptor (TGFBR2) mRNA and protein, and downregulation of TGFBR2 with short hairpin RNA inhibited the invasiveness of GSLCs [71]. Mechanism studies revealed that TGF- $\beta 1$ released by TAM/Ms promoted the expression of MMP-9 by GSLCs, and TGFBR 2 knockdown reduced the invasiveness of these cells in vivo [72-73]. These results demonstrate that TAM/Ms enhance the invasiveness of CD133+ GSLCs via the release of TGF- $\beta 1$, which increases the production of MMP-9 by GSLCs. Therefore, the TGF- $\beta 1$ signaling pathway is a potential therapeutic target for limiting the invasiveness of GSLCs.

Infiltrating leukocytes are responsible for the accumulation of TGF- $\beta 1$ at the invasive front area of tumor, whereas glioma cells are reported to produce TGF- $\beta 2$ [70-71]. Once activated, TGF- $\beta$ binds to the type II TGF- $\beta$ receptor (TGFBR2). The ligand-bound 
TGFBR2 is then able to efficiently trans-activate the type I TGF- $\beta$ receptor (TGFBR1), which transduces intracellular signals through canonical Smaddependent and/or Smad-independent pathways, such as ERK, p38, Rac ,and PI3K-Akt pathways [74-77]. TAM/Ms significantly enhance the invasive capability of GSLCs through paracrine production of TGF- $\beta 1$ and the TGF- $\beta 1$-TGFBR2 signaling pathway. TGFBR2 knockdown was shown to decrease the invasion of GSLCs both in vitro and in vivo.

Furthermore, GSLCs possessed high invasive ability, especially after coculture with TAMs that were derived from transplanted tumors or pretreatment with exogenous TGF- $\beta 1$ [78]. Moreover, recent studies showed that TGF- $\beta$ can increase self-renewal capability of GSLCs $[34,71,77]$. Additionally, TAMs also produce factors such as TGF- $\beta 1$ and IL-10 that may enhance tumor immune suppression and facilitate tumor progression [79]. Whether or not the TGF- $\beta$ signaling pathway contributes to other biological properties of GSLCs, such as invasion, remain to be explored. Development of therapeutic strategies against the TGF- $\beta 1-$ TGFBR2 signaling pathway and exploration of effective means for conversion of tumorinfiltrating macrophages into anti-tumor M1 phenotype may present new countermeasures to disrupt glioma invasion.

\section{CAV1}

Caveolin-1 (CAV1), a plasma membrane molecule with pleiotropic functions, is significantly up-regulated in monocytes in the presence of GBMs [80]. siRNA inhibition of CAV1 restores myeloid cell function, as measured by TNF-alpha secretion, in the presence of GBMs. To determine if a single, dominant molecule was responsible for the GBM-mediated suppression of TAM function, a comprehensive, array-based approach to identify pathways and mechanisms by which GBMs suppress myeloid cell activation was performed. Caveolin-1 (CAV1) was identified as significantly upregulated in myeloid cells in the presence of GBM tumor cells [81]. CAV1 was initially described as a member of a family of scaffolding proteins that interacts with signaling molecules and regulates their activity. It has been reported to have many functions, including the formation of caveolae, membrane trafficking, signal transduction, apoptosis, calcium and lipid homeostasis [82]. More recent evidence indicates that CAV1 also suppresses inflammation [83]. CAV1 expression has been demonstrated in multiple immune cells including monocytes/ macrophages, dendritic cells, and lymphocytes [84]. Similar to the findings in human GBMs, upregulation of CAV1 in murine macrophages dramatically reduced proinflammatory cytokine production (TNF-alpha and IL-6) and increased anti-inflammatory cytokine production (IL-10) [85]. Reported mechanisms of CAV1 mediated immunosuppression in murine models include inhibition of eNOS activity and activation of the MKK3/p38 pathway. Taken together, these data indicate that GBM-mediated suppression of tumorassociated myeloid cell function is mediated at least in part by CAV1, and importantly, that activity can be restored by suppressing CAV1. Currently FDA approved pharmacological inhibitors of CAV1 such as lovastatin and celecoxib may be useful in altering the local tumor microenvironment.

\section{Stat3}

Signal transducer and activator of transcription-3 (Stat3) affects the tumor microenvironment and tumor development by virtue of its association with immunosuppression, angiogenesis, and cancer cell proliferation [86-87]. In some kinds of malignant tumors, including high grade glioma, patients with high Stat 3 activation in tumor cells have significantly worse clinical prognosis [88]. Therefore, Stat3 is thought to be an important target molecule for anticancer therapy, and many researchers have introduced various kinds of Stat3 inhibitors as anticancer drugs [89]. Stat3 signaling in macrophages is known to participate in regulating immune responses. Targeted disruption of Stat 3 signaling resulted in activation of antigen specific $\mathrm{T}$ cells, and suppressed tumor development in murine cancer models [90-92]. In patients with glioblastoma, inhibition of Stat 3 not only suppressed tumor cell growth but also reversed immune tolerance by impairing the immunesuppressive function of alternatively activated macrophage/microglia [93].

M2 macrophages were found to support proliferation of glioma cells through Stat3 activation. Cell-cell interaction during direct contact between tumor cells and macrophages contributes to strong activation of macrophages, which in turn activates tumor cells [94]. In vitro results of the use of a receptor-type tyrosine kinase (RTK) array revealed the importance of macrophage-colony stimulating factor receptor (M-CSFR) activation in this cell-cell interaction. The crucial role of macrophage-colony stimulating factor (M-CSF), especially membrane-type M-CSF (mM- 
CSF), and its binding to M-CSFR during direct cellcell interactions between tumor cells and macrophages was determined [95]. The M-CSFR/Stat3 signals might well prove to be a promising therapy target for patients with high grade glioma.

\section{SDF-1}

A number of tumor-derived chemoattractants, including chemokines and chemokine receptor families, are thought to ensure TAM recruitment. Among the factors that can contribute to the invasive phenotype of glioma cells, stromal-derived factor-1 (SDF-1) has gained increased attention recently. SDF-1 through its receptor, $\mathrm{CXCR} 4$, has been shown to be a major player in tumor metastasis and growth in several human malignancies [96-97]. The SDF-1/CXCR4 axis brings about these effects by recruiting bone marrow derived cells to neovascular niches to promote angiogenesis, as well as stimulating the proliferation and survival of tumor cells in a paracrine manner [38]. A major stimulus for SDF-1 production is hypoxia through the generation of HIF-1. The SDF-1 can in turn enhance the survival of bone marrow-derived mesenchymal stem cells under hypoxic conditions through activation of PI3K/Akt and ERK1/2 pathways [98-99]. SDF-1 in brain tumor invasion provides a new preclinical brain tumor model for designing new treatment options for invasive cases.

\section{CX3CR1}

The chemokine CX3CL1 (also known as fractalkine or neurotactin) exists as a membrane-anchored and potentially soluble form, which suggests that it can exert both localized and systemic effects, and that it may be involved in immune responses that underlie various human diseases [100]. Interestingly, CX3CR1 (also termed RBS11 or V28), the highly specific receptor for CX3CL1, is not only expressed on monocytes, dendritic cells, $\mathrm{T}$ cell subsets, endothelial cells, and platelets, its robust expression has also been described on microglial cells from normal CNS tissue [100-101]. Although a role for CX3CL1/CX3CR1 in tumorigenesis has been established, it was shown in mice that CX3CR1 deficiency does not impact on glioma growth or infiltration of microglia and lymphocytes [102]. Nevertheless, little is known about the expression and function of CX3CR 1 in human glioma-infiltrating microglia/macrophages (GIMs), in part because of the scarcity of fresh human glioma tissue and the technical difficulties in obtaining pure human GIMs from CNS tissue [103]. CX3CR1 was overexpressed in gliomas at both the mRNA and protein level and was expressed in GIMs in situ. As revealed by co-localization of $\mathrm{CX} 3 \mathrm{CR} 1$ with ionized calcium-binding adapter molecule 1 (Iba1) and CD11b/ c, CX3CR1 was exclusively found to be expressed on GIMs [104]. Moreover, following enrichment of GIMs from fresh human glioblastoma samples, it has been showed that CX3CL1 promoted GIMs adhesion and migration in vitro [105]. Finally, based on the CX3CL1-induced up-regulation of their expression, it has been identified that the matrix metalloproteases (MMPs) 2, 9, and 14 act as potential candidates facilitating these properties.

\section{MIF}

Macrophage migration inhibitory factor (MIF) is currently emerging as a key factor in glioma [18]. For example, its expression correlates well with glioma recurrence and poorer prognosis and, thereby, MIF is currently being considered as a valuable and independent prognostic indicator for patients with glioma [106]. MIF is also expressed by a variety of immune cells, including macrophages, lymphocytes, and eosinophils, as well as by endothelial cells and epithelial cells. Further, MIF has been shown to promote tumor progression in many types of malignancies and been implicated as a direct link between the process of inflammation and tumor growth, thus making it a potential target for anti-cancer treatment [107].

\section{Multifunctional nanoparticles targeting TAMs for glioma therapy}

Despite tremendous efforts to develop diagnostic tools and therapeutic avenues, the treatment of glioma remains a formidable challenge in the field of neurooncology [108]. Chemotherapy for intracranial gliomas is hampered by he complexity of the brain and limited delivery of therapeutic agents through the blood brain barrier (BBB) [109]. The BBB maintains the homeostasis of the highly sensitive central nervous system (CNS) and protects the brain from neurotoxic substances prevalent in the peripheral circulatory system. This comprises of endothelial cells that does not allow entry of exogenous material, bacteria, viruses and chemotherapeutic agents. However, BBB allows the passage of small sized particles that are hydrophobic in nature [110]. An optimal therapeutic agent for brain tumors would selectively cross the 
$\mathrm{BBB}$, accumulates at the site of a tumor, preventing adequate destruction of malignant cells and be activated from an innocuous prodrug within the tumor.

The field of nanomedicine has opened opportunities for improved brain cancer diagnosis and treatment. By using a nanometer sized delivery system, a significant drug loading per unit volume can be achieved, which is of crucial importance when high dosing is required [111]. With the rapid development of nanotechnology for biomedical applications, multifunctional nanoparticles offer the potential to improve upon many of these issues and may lead to breakthroughs in brain tumor management. Multifunctional nanoparticles containing optical, thermal, and magnetic properties are promising systems that offer new opportunities to overcome the limitations of current brain tumor management options in the clinic [108]. Polymeric nanoparticles are amongst the most preferred delivery system for treatment of cancers due to higher penetrability, sustainability, degradability and better payload. Chemotherapy-loaded nanoparticles have resulted in sustained release formulations that can lower systemic toxicity and produce greater antitumor effects. The field has been moving at a rapid pace, enabling nanoparticles to be utilized in recent clinical trials [112]. Small sized particles are better permeated though the barrier with the target ability to the TAMs cells. While not exhaustive, the list of nanoparticles being used in the treatment of experimental GBM includes polymeric particles, micelles, nanoshells, quantum dots, and magnetic iron-oxide nanoparticles (IONPs). Nanotubes are another formulation of nanoparticle, being used to create structures that can trap diagnostic or therapeutic modalities within a cage [108, 109].

BLZ945 has high affinity and specificity for colonystimulating factor 1 receptor (CSF-1R), specifically reduced macrophage proliferation and survival in vitro without affecting glioma cell viability [13]. BLZ945 may be incorporated into the nanoparticle structure for therapy targeting TAM. Amphotericin (AmpB) B has been validated as an activator of monocytoid cells and found that $A m p B$ enhanced the microglial reduction of BTIC (Brain tumor initiating cells spheres. It has been noted that monocytes and microglia from glioblastoma patients were unable to reduce BTIC sphere formation until they received AmpB [113]. It can be formed into stable nanometer-sized prodrugs that inhibit the growth of BTIC, suggesting its potential application as anticancer agent. The nitrogen mustard cyclophosphamide induces an acute secretory activating phenotype (ASAP), releasing CCL4, IL8, VEGF, and TNFa which induce macrophage infiltration and phagocytic activity in the bone marrow [114]. A liposomal encapsulation of the alemtuzumab and CTX (cyclophosphamide) is another delivery system resulted in a dramatic synergistic effect in tumor microenvironments. Despite the tremendous efforts thus far, only a few nanoparticle systems have been approved for clinic trials, indicating that many obstacles still need to be overcome in order to make these freely available for clinical treatment. Phase III studies will need to be undertaken to prove their effectiveness. Nanotechnology has quickly become a very promising tool in the ongoing research to tackle the difficulties in treating GBM.

\section{Conclusion}

Tumor-associated monocytes/microglia in malignant gliomas seem to be a dreadful enemy, resulting in an altered activation state within the local tumor microenvironment characterized by upregulation of potent immunosuppressive mediators, deficiencies in antitumor effector functions, and participation in paracrine tumorigenic signaling loops involving expression of trophic factors and their cognate receptors (summarized in Figure 1). Although the role of different cell types that constitute tumor microenvironment of glioma remain unclear during tumor progression, a turning point seems to occur late in tumor development, perhaps providing a better opportunity for clinically based immunotherapy. Given the compelling evidence that TAMs contribute significantly to the creation and maintenance of immunosuppression and tumor progression, it is unlikely that clinically effective immunotherapy against malignant gliomas will be achieved until we gain a better understanding of how to influence TAM function in the local tumor microenvironment. Nanoprodrug may also play a significant auxiliary role in the immunotherapy towards glioma while traditional therapeutic agents are difficult to be delivered through the blood brain barrier (BBB).

\section{Acknowledgements}

This work was supported by National Natural Science Foundation of China (Nos. 81172398 and 81272801) and 


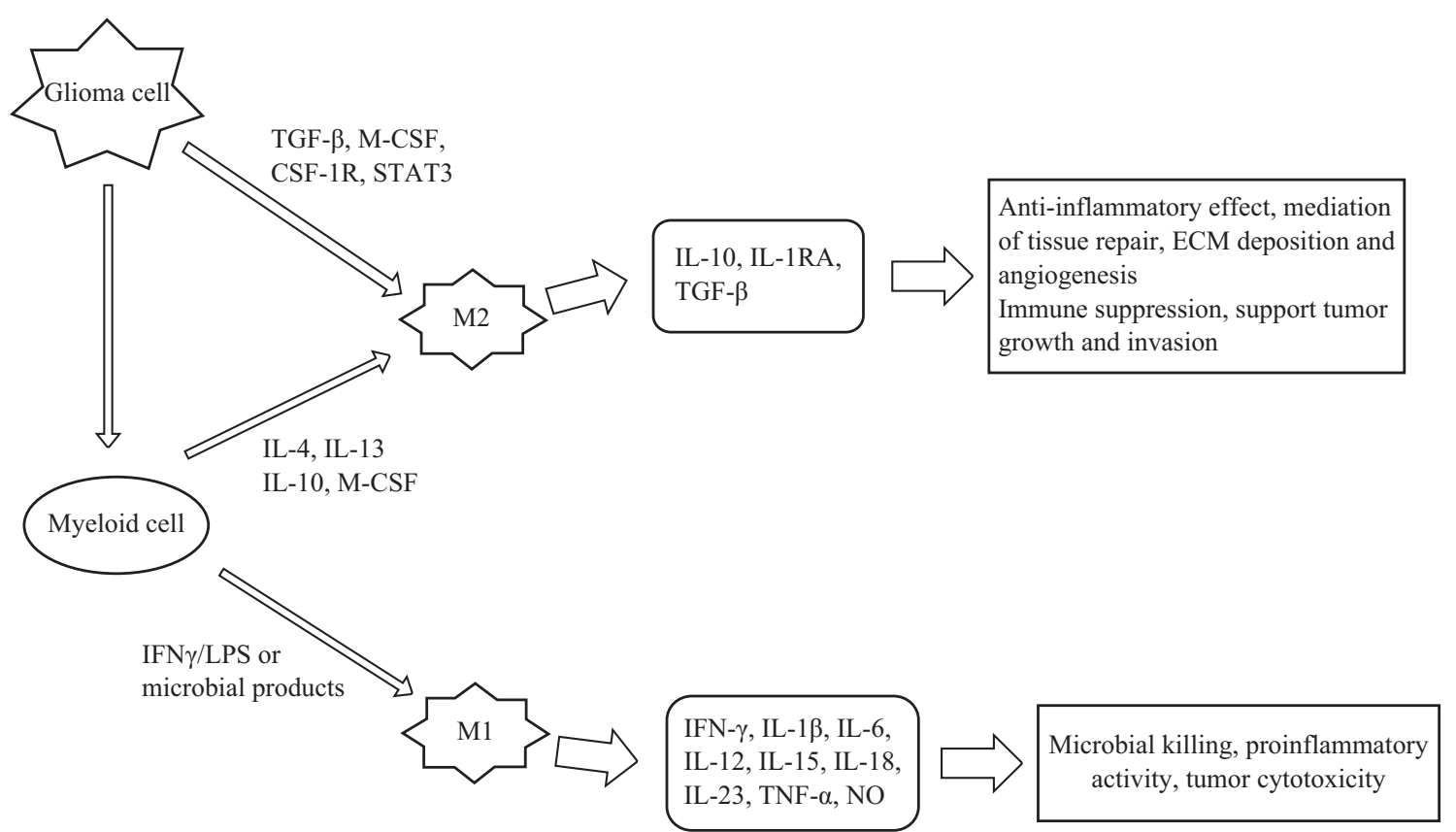

Fig. 1 Microglia in glioma can polarized into two macrophage subpopulations: M1 (classically activated macrophages) and M2 (alternatively activated macrophages). When mononuclear/phagocytic cells are stimulated by pathogens and pro-inflammatory molecules, they differentiate into the M1 phenotype, resulting in killing microorganisms and tumor cells through production of proinflammatory mediators (such as TNF- $\alpha$, IL-1 $\beta$, and IL-12). When mononuclear/phagocytic cells are activated by antiinflammatory molecules such as IL-4 and IL-10, they differentiate into the M2 phenotype and have been shown to promote cancer by secreting proangiogenic factors and anti-inflammatory effect. Tumor-derived molecules, such as TGF- $\beta$ and M-CSF, can induce glioma-infiltrating microglia/microphages toward the M2 phenotype and accordingly stimulate the production of anti-inflammatory molecules.

National Key Basic Research Program of China (“973” Project) (2010CB933901).

\section{References}

[1] Kohanbash G, McKaveney K, Sakaki M, et al. GMCSF Promotes the Immunosuppressive Activity of Glioma-Infiltrating Myeloid Cells through Interleukin-4 Receptor- $\alpha$. Cancer research, 2013, 73(21): 6413-6423.

[2] Okada H, Kohanbash G, Zhu X, et al. Immunotherapeutic approaches for glioma. Critical Reviews ${ }^{\mathrm{TM}}$ in Immunology, 2009, 29(1): 1-42.

[3] Tran B, Rosenthal M A. Survival comparison between glioblastoma multiforme and other incurable cancers. Journal of Clinical Neuroscience, 2010, 17(4): 417-421.

[4] Goodenberger M K L, Jenkins R B. Genetics of adult glioma. Cancer genetics, 2012, 205(12): 613-621.

[5] Vredenburgh J J, Desjardins A, Reardon D A, et al. Experience with irinotecan for the treatment of malignant glioma. Neuro-oncology, 2009, 11(1): 80-91.

[6] Anton K, Baehring J M, Mayer T. Glioblastoma multiforme: overview of current treatment and future perspectives. Hematology/oncology clinics of North America, 2012, 26(4): 825-853.

[7] Stupp R, Hegi M E, Mason W P, et al. Effects of radiotherapy with concomitant and adjuvant temozolomide versus radiotherapy alone on survival in glioblastoma in a randomised phase III study: 5-year analysis of the EORTC-NCIC trial. The lancet oncology, 2009, 10(5): 459-466.

[8] Poli A, Wang J, Domingues O, et al. Targeting glioblastoma with NK cells and mAb against NG2/ CSPG4 prolongs animal survival. Oncotarget, 2013, 4(9): 1527.

[9] Ye X, Xu S, Xin Y, et al. Tumor-associated microglia/ macrophages enhance the invasion of glioma stemlike cells via TGF- $\beta 1$ signaling pathway. The Journal of Immunology, 2012, 189(1): 444-453.

[10] Zagzag D, Salnikow K, Chiriboga L, et al. Downregulation of major histocompatibility complex antigens in invading glioma cells: stealth invasion of the brain. Laboratory investigation, 2005, 85(3): 328-341.

[11] Hussain S F, Yang D, Suki D, et al. The role of human glioma-infiltrating microglia/macrophages in mediating antitumor immune responses. Neuro-oncology, 2006, 8(3): 261-279.

[12] Okada H, Kalinski P, Ueda R, et al. Induction of CD8+ T-cell responses against novel glioma-associated antigen peptides and clinical activity by vaccinations with $\alpha$-type 1 polarized dendritic cells and polyinosinic-polycytidylic acid stabilized by lysine and carboxymethylcellulose in patients with recurrent malignant glioma. Journal of Clinical Oncology, 2011, 29(3): 330-336.

[13] Pyonteck S M, Akkari L, Schuhmacher A J, et al. CSF$1 \mathrm{R}$ inhibition alters macrophage polarization and blocks glioma progression. Nature medicine, 2013, 19(10): 1264-1272.

[14] Wen P Y, Kesari S. Malignant gliomas in adults. New England Journal of Medicine, 2008, 359(5): 492-507.

[15] Dunn G P, Rinne M L, Wykosky J, et al. Emerging insights into the molecular and cellular basis of glioblastoma. Genes \& development, 2012, 26(8): 756784.

[16] Balkwill F R, Mantovani A. Cancer-related inflammation: common themes and therapeutic opportunities.Seminars 
in cancer biology. Academic Press, 2012, 22(1): 33-40.

[17] Joyce J A, Pollard J W. Microenvironmental regulation of metastasis[J]. Nature Reviews Cancer, 2009, 9(4): 239 252.

[18] Põlajeva J, Bergström T, Edqvist P H, et al. Gliomaderived macrophage migration inhibitory factor (MIF) promotes mast cell recruitment in a STAT5-dependent manner. Molecular oncology, 2013, 8(1): 50-58.

[19] Hanahan D, Coussens L M. Accessories to the crime: functions of cells recruited to the tumor microenvironment. Cancer cell, 2012, 21(3): 309-322.

[20] Arismendi-Morillo G, Castellano-Ramírez A, Medina Z. Ultrastructural characterization of macrophage-like mononuclear leukocytes in human astrocytic tumors. Ultrastructural pathology, 2010, 34(6): 321-326.

[21] Nyberg P, Salo T, Kalluri R. Tumor microenvironment and angiogenesis. Frontiers in bioscience: a journal and virtual library, 2007, 13: 6537-6553.

[22] [22] Sasaki A, Yokoo H, Tanaka Y, et al. Characterization of microglia/macrophages in gliomas developed in S$100 \beta$-v-erbB transgenic rats. Neuropathology, 2013 33(5): 505-514.

[23] Graeber M B, Streit W J. Microglia: biology and pathology. Acta neuropathologica, 2010, 119(1): 89-105.

[24] Garris C, Pittet M J. Therapeutically reeducating macrophages to treat GBM. Nature medicine, 2013, 19(10): 1207-1208.

[25] Wu A, Wei J, Kong L Y, et al. Glioma cancer stem cells induce immunosuppressive macrophages/microglia. Neuro-oncology, 2010, 12(11): 1113-1125.

[26] Streit W J, Kreutzberg G W. Response of endogenous glial cells to motor neuron degeneration induced by toxic ricin. Journal of Comparative Neurology, 1988, 268(2): 248-263.

[27] Kostianovsky A M, Maier L M, Anderson R C, et al. Astrocytic regulation of human monocytic/microglial activation. The Journal of Immunology, 2008, 181(8): 5425-5432.

[28] van Rossum D, Hanisch U K. Microglia. Metabolic brain disease, 2004, 19(3-4): 393-411.

[29] Kennedy B C, Showers C R, Anderson D E, et al. Tumorassociated macrophages in glioma: friend or foe? Journal of oncology, 2013, 2013:486912.

[30] MacMicking J, Xie Q, Nathan C. Nitric oxide and macrophage function. Annual review of immunology, 1997, 15(1): 323-350

[31] Boehm U, Klamp T, Groot M, and Howard J C. Cellular responses to interferon-gamma. Annual Review of Immunology, 1997, 15:749-795,

[32] Hussain S F, Yang D, Suki D, et al. Innate immune functions of microglia isolated from human glioma patients. Journal of translational medicine, 2006, 4(1): 15.

[33] Sliwa M, Markovic D, Gabrusiewicz K, et al. The invasion promoting effect of microglia on glioblastoma cells is inhibited by cyclosporin A. Brain, 2007, 130(2): 476-489.

[34] Wesolowska A, Kwiatkowska A, Slomnicki L, et al. Microglia-derived TGF- $\beta$ as an important regulator of glioblastoma invasion-an inhibition of TGF- $\beta$ dependent effects by shRNA against human TGF- $\beta$ type II receptor. Oncogene, 2008, 27(7): 918-930.

[35] Voronov E, Shouval D S, Krelin Y, et al. IL-1 is required for tumor invasiveness and angiogenesis. Proceedings of the National Academy of Sciences, 2003, 100(5): 2645 2650.

[36] Hiratsuka S, Nakamura K, Iwai S, et al. MMP9 induction by vascular endothelial growth factor receptor- 1 is involved in lung-specific metastasis. Cancer cell, 2002, 2(4): $289-300$
[37] Komohara Y, Ohnishi K, Kuratsu J, et al. Possible involvement of the M2 anti-inflammatory macrophage phenotype in growth of human gliomas. The Journal of pathology, 2008, 216(1): 15-24.

[38] Wang S C, Hong J H, Hsueh C, et al. Tumor-secreted SDF-1 promotes glioma invasiveness and TAM tropism toward hypoxia in a murine astrocytoma model. Laboratory Investigation, 2012, 92(1): 151-162.

[39] Badie B, Schartner J M. Flow cytometric characterization of tumor-associated macrophages in experimental gliomas. Neurosurgery, 2000, 46(4): 957-962.

[40] Kennedy B C, Maier L M, D'Amico R, et al. Dynamics of central and peripheral immunomodulation in a murine glioma model. BMC immunology, 2009, 10(1): 11.

[41] $\mathrm{Li} \mathrm{W}$, Graeber M B. The molecular profile of microglia under the influence of glioma. Neuro-oncology, 2012, 14(8): 958-978.

[42] Filipazzi P, Huber V, Rivoltini L. Phenotype, function and clinical implications of myeloid-derived suppressor cells in cancer patients. Cancer Immunology, Immunotherapy, 2012, 61(2): 255-263.

[43] Rodrigues J C, Gonzalez G C, Zhang L, et al. Normal human monocytes exposed to glioma cells acquire myeloid-derived suppressor cell-like properties. Neurooncology, 2010, 12(4): 351-365.

[44] Huettner C, Czub S, Kerkau S, et al. Interleukin 10 is expressed in human gliomas in vivo and increases glioma cell proliferation and motility in vitro. Anticancer research, 1996, 17(5A): 3217-3224.

[45] Badie B, Schartner J, Prabakaran S, et al. Expression of Fas ligand by microglia: possible role in glioma immune evasion. Journal of neuroimmunology, 2001, 120(1): 1924.

[46] Didenko V V, Ngo H N, Minchew C, et al. Apoptosis of $\mathrm{T}$ lymphocytes invading glioblastomas multiforme: a possible tumor defense mechanism. Journal of neurosurgery, 2002, 96(3): 580.

[47] Ford A L, Foulcher E, Lemckert F A, et al. Microglia induce CD4 T lymphocyte final effector function and death. The Journal of experimental medicine, 1996, 184(5): 1737-1745.

[48] Jansen T, Tyler B, Mankowski J L, et al. FasL gene knock-down therapy enhances the antiglioma immune response. Neuro-oncology, 2010, 12(5): 482-489.

[49] Mitchell D A, Fecci P E, Sampson J H. Immunotherapy of malignant brain tumors. Immunological reviews, 2008, 222(1): 70-100.

[50] Wintterle S, Schreiner B, Mitsdoerffer M, et al. Expression of the B7-Related Molecule B7-H1 by Glioma Cells A Potential Mechanism of Immune Paralysis. Cancer research, 2003, 63(21): 7462-7467.

[51] Kjellman C, Olofsson S P, Hansson O, et al. Expression of TGF- $\beta$ isoforms, TGF- $\beta$ receptors, and SMAD molecules at different stages of human glioma. International Journal of Cancer, 2000, 89(3): 251-258.

[52] Germain D, Frank D A. Targeting the cytoplasmic and nuclear functions of signal transducers and activators of transcription 3 for cancer therapy. Clinical Cancer Research, 2007, 13(19): 5665-5669.

[53] Schartner J M, Hagar A R, Van Handel M, et al. Impaired capacity for upregulation of MHC class II in tumorassociated microglia. Glia, 2005, 51(4): 279-285.

[54] Samuels V, Barrett J M, Bockman S, et al. Immunocytochemical study of transforming growth factor expression in benign and malignant gliomas. The American journal of pathology, 1989, 134(4): 894.

[55] Badie B, Schartner J, Klaver J, et al. In vitro modulation of microglia motility by glioma cells is mediated by hepatocyte growth factor/scatter factor. Neurosurgery, 1999, 44(5): 1077-1082. 
[56] Tsai J C, Goldman C K, Gillespie G Y. Vascular endothelial growth factor in human glioma cell lines: induced secretion by EGF, PDGF-BB, and bFGF. Journal of neurosurgery, 1995, 82(5): 864-873.

[57] Carmeliet P. VEGF as a key mediator of angiogenesis in cancer. Oncology, 2005, 69(Suppl. 3): 4-10.

[58] Shibuya M, Claesson-Welsh L. Signal transduction by VEGF receptors in regulation of angiogenesis and lymphangiogenesis. Experimental cell research, 2006, 312(5): 549-560.

[59] Waltenberger J, Claesson-Welsh L, Siegbahn A, et al. Different signal transduction properties of KDR and Flt1, two receptors for vascular endothelial growth factor. Journal of Biological Chemistry, 1994, 269(43): 2698826995.

[60] Alitalo K, Carmeliet P. Molecular mechanisms of lymphangiogenesis in health and disease. Cancer cell, 2002, 1(3): 219-227.

[61] Kerber M, Reiss Y, Wickersheim A, et al. Flt-1 signaling in macrophages promotes glioma growth in vivo. Cancer Research, 2008, 68(18): 7342-7351.

[62] Roggendorf W, Strupp S, Paulus W. Distribution and characterization of microglia/macrophages in human brain tumors. Acta neuropathologica, 1996, 92(3): 288293.

[63] Hattori K, Dias S, Heissig B, et al. Vascular endothelial growth factor and angiopoietin-1 stimulate postnatal hematopoiesis by recruitment of vasculogenic and hematopoietic stem cells. The Journal of experimental medicine, 2001, 193(9): 1005-1014.

[64] Murakami M, Iwai S, Hiratsuka S, et al. Signaling of vascular endothelial growth factor receptor-1 tyrosine kinase promotes rheumatoid arthritis through activation of monocytes/macrophages. Blood, 2006, 108(6): 18491856.

[65] Oura H, Bertoncini J, Velasco P, et al. A critical role of placental growth factor in the induction of inflammation and edema formation. Blood, 2003, 101(2): 560-567.

[66] Detmar M, Brown L F, Schön M P, et al. Increased microvascular density and enhanced leukocyte rolling and adhesion in the skin of VEGF transgenic mice. Journal of Investigative Dermatology, 1998, 111(1): 1-6.

[67] Barbera-Guillem E, Nyhus J K, Wolford C C, et al. Vascular endothelial growth factor secretion by tumorinfiltrating macrophages essentially supports tumor angiogenesis, and IgG immune complexes potentiate the process. Cancer research, 2002, 62(23): 7042-7049.

[68] Patel S, Player M R. Colony-stimulating factor-1 receptor inhibitors for the treatment of cancer and inflammatory disease. Current topics in medicinal chemistry, 2009, 9(7): 599-610.

[69] Ruffell B, Affara N I, Coussens L M. Differential macrophage programming in the tumor microenvironment. Trends in immunology, 2012, 33(3): 119-126.

[70] Bruna A, Darken R S, Rojo F, et al. High TGF $\beta$-Smad activity confers poor prognosis in glioma patients and promotes cell proliferation depending on the methylation of the PDGF-B gene. Cancer cell, 2007, 11(2): 147-160.

[71] Peñuelas S, Anido J, Prieto-Sánchez R M, et al. TGF- $\beta$ increases glioma-initiating cell self-renewal through the induction of LIF in human glioblastoma. Cancer cell, 2009, 15(4): 315-327.

[72] Kim H S, Luo L, Pflugfelder S C, et al. Doxycycline inhibits TGF- $\beta 1$-induced MMP-9 via Smad and MAPK pathways in human corneal epithelial cells. Investigative ophthalmology \& visual science, 2005, 46(3): 840-848.

[73] Chen D, Ping Y, Yu S, et al. Downregulating FPR restrains xenograft tumors by impairing the angiogenic potential and invasive capability of malignant glioma cells. Biochemical and biophysical research communications, 2009, 381(3): 448-452.

[74] Massagué J. TGF $\beta$ in cancer. Cell, 2008, 134(2): 215230.

[75] Bierie B, Moses H L. Transforming growth factor beta (TGF- $\beta$ ) and inflammation in cancer. Cytokine \& growth factor reviews, 2010, 21(1): 49-59.

[76] Zhang Y E. Non-Smad pathways in TGF- $\beta$ signaling. Cell research, 2009, 19(1): 128-139.

[77] Ikushima H, Todo T, Ino $Y$, et al. Autocrine TGF- $\beta$ signaling maintains tumorigenicity of glioma-initiating cells through Sry-related HMG-box factors. Cell stem cell, 2009, 5(5): 504-514.

[78] Yu S, Ping Y, Yi L, et al. Isolation and characterization of cancer stem cells from a human glioblastoma cell line U87. Cancer letters, 2008, 265(1): 124-134.

[79] Mantovani A, Schioppa T, Porta C, et al. Role of tumorassociated macrophages in tumor progression and invasion. Cancer and Metastasis Reviews, 2006, 25(3): 315-322.

[80] Shimato S, Anderson L M, Asslaber M, et al. Inhibition of Caveolin-1 Restores Myeloid Cell Function in Human Glioblastoma. PloS one, 2013, 8(10): e77397.

[81] Wang X M, Kim H P, Song R, et al. Caveolin-1 confers antiinflammatory effects in murine macrophages via the MKK3/p38 MAPK pathway. American journal of respiratory cell and molecular biology, 2006, 34(4): 434.

[82] Anderson R G. Caveolae: where incoming and outgoing messengers meet. Proceedings of the National Academy of Sciences, 1993, 90(23): 10909-10913.

[83] de Almeida C J, Witkiewicz A K, Jasmin J F, et al. Caveolin-2-deficient mice show increased sensitivity to endotoxemia. Cell Cycle, 2011, 10(13): 2151-2161.

[84] Harris J, Werling D, Hope J C, et al. Caveolae and caveolin in immune cells: distribution and functions. Trends in immunology, 2002, 23(3): 158-164.

[85] Santizo R A, Xu H L, Galea E, et al. Combined endothelial nitric oxide synthase upregulation and caveolin-1 downregulation decrease leukocyte adhesion in pial venules of ovariectomized female rats. Stroke, 2002, 33(2): 613-616.

[86] Yu H, Kortylewski M, Pardoll D. Crosstalk between cancer and immune cells: role of STAT3 in the tumour microenvironment. Nature Reviews Immunology, 2007, 7(1): 41-51.

[87] Yoshimura A. Signal transduction of inflammatory cytokines and tumor development. Cancer science, 2006, 97(6): 439-447.

[88] Abou-Ghazal M, Yang D S, Qiao W, et al. The incidence, correlation with tumor-infiltrating inflammation, and prognosis of phosphorylated STAT3 expression in human gliomas. Clinical Cancer Research, 2008, 14(24): 82288235.

[89] Page B D G, Ball D P, Gunning P T. Signal transducer and activator of transcription 3 inhibitors: a patent review. Expert opinion on therapeutic patents, 2011, 21(1): 65-83.

[90] Cheng F, Wang H W, Cuenca A, et al. A critical role for Stat3 signaling in immune tolerance. Immunity, 2003, 19(3): 425-436.

[91] Wu L, Du H, Li Y, et al. Signal transducer and activator of transcription 3 (Stat3C) promotes myeloid-derived suppressor cell expansion and immune suppression during lung tumorigenesis. The American journal of pathology, 2011, 179(4): 2131-2141.

[92] Fujita M, Kohanbash G, Fellows-Mayle W, et al. COX2 blockade suppresses gliomagenesis by inhibiting myeloid-derived suppressor cells. Cancer research, 2011, 71(7): 2664-2674.

[93] Hussain S F, Kong L Y, Jordan J, et al. A novel small 
molecule inhibitor of signal transducers and activators of transcription 3 reverses immune tolerance in malignant glioma patients. Cancer research, 2007, 67(20): 96309636.

[94] Komohara Y, Horlad H, Ohnishi K, et al. Importance of direct macrophage - Tumor cell interaction on progression of human glioma. Cancer science, 2012, 103(12): 2165-2172.

[95] Komohara Y, Horlad H, Ohnishi K, et al. M2 macrophage/microglial cells induce activation of Stat3 in primary central nervous system lymphoma. J Clin Exp Hematop, 2011, 51(2): 93-99.

[96] Balkwill F. Chemokine biology in cancer. Seminars in immunology. Academic Press, 2003, 15(1): 49-55.

[97] Dewan M Z, Ahmed S, Iwasaki Y, et al. Stromal cellderived factor- 1 and CXCR4 receptor interaction in tumor growth and metastasis of breast cancer. Biomedicine \& pharmacotherapy, 2006, 60(6): 273-276.

[98] Aghi M, Cohen K S, Klein R J, et al. Tumor stromalderived factor-1 recruits vascular progenitors to mitotic neovasculature, where microenvironment influences their differentiated phenotypes. Cancer research, 2006, 66(18): 9054-9064.

[99] Bazan J F, Bacon K B, Hardiman G, et al. A new class of membrane-bound chemokine with a $\mathrm{CX} 3 \mathrm{C}$ motif $[\mathrm{J}]$. 1997, 385(6617):640-4.

[100] Held-Feindt J, Hattermann K, Müerköster S S, et al. CX3CR1 promotes recruitment of human gliomainfiltrating microglia/macrophages (GIMs). Experimental cell research, 2010, 316(9): 1553-1566.

[101] Imai T, Hieshima K, Haskell C, et al. Identification and Molecular Characterization of Fractalkine Receptor CX3CR1, which Mediates Both Leukocyte Migration and Adhesion. Cell, 1997, 91(4): 521-530.

[102] Rodero M, Marie Y, Coudert M, et al. Polymorphism in the microglial cell-mobilizing CX3CR1 gene is associated with survival in patients with glioblastoma. Journal of Clinical Oncology, 2008, 26(36): 5957-5964.

[103] Xin H, Kikuchi T, Andarini S, et al. Antitumor immune response by CX3CL1 fractalkine gene transfer depends on both NK and T cells. European journal of immunology, 2005, 35(5): 1371-1380.

[104] Somasundaram R, Herlyn D. Chemokines and the microenvironment in neuroectodermal tumor-host interaction. Seminars in cancer biology. Academic Press,
2009, 19(2): 92-96.

[105] Yu Y R A, Fong A M, Combadiere C, et al. Defective antitumor responses in CX3CR1 - deficient mice. International journal of cancer, 2007, 121(2): 316-322.

[106] Wang X, Tian X, Li Y, et al. Elevated expression of macrophage migration inhibitory factor correlates with tumor recurrence and poor prognosis of patients with gliomas. Journal of neuro-oncology, 2012, 106(1): 43-51.

[107] Conroy H, Mawhinney L, Donnelly S C. Inflammation and cancer: macrophage migration inhibitory factor (MIF) — the potential missing link. QJM, 2010, 103(11): 831836.

[108] Cheng Y, Morshed R A, Auffinger B, et al. Multifunctional nanoparticles for brain tumor imaging and therapy. Advanced drug delivery reviews, 2014,66C: 42-57.

[109] Groothuis D R. The blood-brain and blood-tumor barriers: a review of strategies for increasing drug delivery. Neuro-oncology, 2000, 2(1): 45-59.

[110] Begley D J, Brightman M W. Structural and functional aspects of the blood-brain barrier. Peptide transport and delivery into the central nervous system. Birkhäuser Basel, 2003: 39-78.

[111] Lee B S, Yoon C W, Osipov A, et al. Nanoprodrugs of NSAIDs: Preparation and characterization of flufenamic acid nanoprodrugs. Journal of drug delivery, 2011, 2011: 980720.

[112] Nduom E K, Bouras A, Kaluzova M, et al. Nanotechnology applications for glioblastoma. Neurosurgery Clinics of North America, 2012, 23(3): 439-449.

[113] Sarkar S, Döring A, Zemp F J, et al. Therapeutic activation of macrophages and microglia to suppress brain tumor-initiating cells. Nature neuroscience, 2014, 17(1): 46-55.

[114] Pallasch C P, Leskov I, Braun C J, et al. Sensitizing Protective Tumor Microenvironments to AntibodyMediated Therapy. Cell, 2014, 156(3): 590-602.

Copyright $\subseteq 2014$ Xian-Bin Lin, Guo-Han Hu, Hua-Lin Fu and Wei-Lin Jin. This is an open-access article distributed under the terms of the Creative Commons Attribution License, which permits unrestricted use, distribution, and reproduction in any medium, provided the original author and source are credited. 